\title{
Joint routing and scheduling optimization in arbitrary ad hoc networks: Comparison of cooperative and hop-by-hop forwarding
}

\author{
Antonio Capone, Stefano Gualandi and Di Yuan
}

\section{Linköping University Post Print}

N.B.: When citing this work, cite the original article.

Original Publication:

Antonio Capone, Stefano Gualandi and Di Yuan, Joint routing and scheduling optimization in arbitrary ad hoc networks: Comparison of cooperative and hop-by-hop forwarding, 2011, Ad hoc networks, (9), 7, 1256-1269.

http://dx.doi.org/10.1016/j.adhoc.2011.02.002

Copyright: Elsevier Science B. V., Amsterdam http://www.elsevier.com/

Postprint available at: Linköping University Electronic Press

http://urn.kb.se/resolve?urn=urn:nbn:se:liu:diva-69767 


\title{
Joint Routing and Scheduling Optimization in Arbitrary Ad Hoc Networks: Comparison of Cooperative and Hop-by-Hop Forwarding
}

\author{
Antonio Capone $^{\mathrm{a}}$, Stefano Gualandi ${ }^{\mathrm{a}}$, Di Yuan ${ }^{\mathrm{b}}$ \\ ${ }^{a}$ Dipartimento di Elettronica e Informatica, Politecnico di Milano, \\ Viale Ponzio 24/A, 20133, Milan, Italy \\ ${ }^{b}$ Department of Science and Technology, Linköping Institute of Technology, \\ SE-601 74 Norrköping, Sweden
}

\begin{abstract}
Cooperation schemes form a key aspect of infrastructure-less wireless networks that allow nodes that cannot directly communicate to exchange information through the help of intermediate nodes. The most widely adopted approach is based on hop-by-hop forwarding at the network layer along a path to destination. Cooperative relaying brings cooperation to the physical layer in order to fully exploit wireless resources. The concept exploits channel diversity by using multiple radio units to transmit the same message. The underlying fundamentals of cooperative relaying have been quite well-studied from a transmission efficiency point of view, in particular with a single pair of source and destination. Results of its performance gain in a multi-hop networking context with multiple sources and destinations are, however, less available. In this paper, we provide an optimization approach to assess the performance gain of cooperative relaying vis-a-vis conventional multi-hop forwarding under arbitrary network topology. The approach joint optimizes packet routing and transmission scheduling, and generalizes classical optimization schemes for non-cooperative networks. We provide numerical results demonstrating that the gain of cooperative relaying in networking scenarios is in general rather small and decreases when network
\end{abstract}

Email addresses: capone@elet.polimi.it (Antonio Capone), gualandi@elet.polimi.it (Stefano Gualandi), diyua@itn.liu.se (Di Yuan)

Preprint submitted to Ad Hoc Networks

December 30, 2010 
connectivity and the number of traffic flows increase, due to interference and resource reuse limitations. In addition to quantifying the performance gain, our approach leads to a new framework for optimizing routing and scheduling in cooperative networks under a generalized Spacial Time Division Multiple Access (STDMA) scheme.

Keywords: Cooperative Networking, Routing and Scheduling, Column Generation

\section{Introduction}

Infrastructure-less wireless networks have received much attention from the research community and generated a plethora of applications (including ad hoc, sensor, and mesh networks). In most of these application scenarios, direct communication is not possible for all node pairs due to the transmission range limitations, and thus cooperative approaches are adopted. The goal is to allow nodes that cannot directly communicate to exchange information through intermediate nodes that relay messages towards their destinations.

The first and most common cooperation mechanism is based on hop-by-hop forwarding along a path composed by a sequence of nodes from the source to the destination. At each hop, a single node forwards an information packet to the next node in the path which is usually dynamically selected through a routing protocol [1]. This conventional multi-hop forwarding scheme has been adopted in almost all practical implementations of ad hoc, sensor and wireless mesh networks, and analyzed by a huge amount of literature.

Recently, in order to overcome the capacity limits of conventional multihop forwarding, a new approach exploiting cooperation also at the transmission layer has been considered $[2,3]$. The idea, that dates back to the work of van der Meulen [4] and Cover [5], is to use multiple relays whose transmissions are combined together at the receiver. Exploiting the broadcast nature of the radio channel, transmissions can be received and then simultaneously forwarded by groups of nodes, each acting as a virtual array of antennas $[6,7]$. Relay nodes 
can decode-and-forward or just amplify-and-forward information packets [8, 9].

Similarly to multiple antenna systems, with cooperative relaying there are some remarkable advantages in terms of channel capacity [10-12]. However, whereas the performance gain of cooperative relaying is easy to understand in single source-destination scenarios, the case of multiple sources and destinations is more complex to analyze $[21,22]$ and requires the design of cooperation protocols for multiple access, channel state information, and routing [23-25]. In general, it is expected that the signaling overhead for cooperation information is higher than traditional hop-by-hop forwarding. For some specific scenarios, it has been shown that protocols for cooperation may require twice the bandwidth in comparison to non-cooperative schemes [8]. However, protocol overhead is not the only limiting factor of the performance of cooperative relaying. In this paper we aim to show that, even neglecting the impact of increased protocol overhead, from a network capacity perspective with multiple sources and destinations, the potential gain of cooperative relaying may be quite small. This is due to the interference generated by simultaneous transmissions and the limitations caused by half-duplex operation of wireless devices.

Indeed, from a network point of view, in addition to radio channel capacity, there is another important element that determines the overall efficiency: the reuse level of radio resources in the network. Parallel transmissions of nodes are possible if they are sufficiently apart and the interference at their receivers is low enough to allow for correct decoding of information packets. We argue that, even if cooperative relaying can increase the radio channel capacity, or equivalently the transmission range, it may negatively affect the reuse level. Transmissions from multiple relays generate interference that adds to the noise, thus limiting the number of parallel transmissions of different packets. Moreover, since nodes are usually equipped with a single transceiver, at a given time they can be involved in only one cooperation group for transmitting or receiving a packet, further limiting resource reuse. In the extreme case, all nodes cooperate to relay the packet of a source-destination pair, and no parallel transmission can occur in the network (Figure 1). 


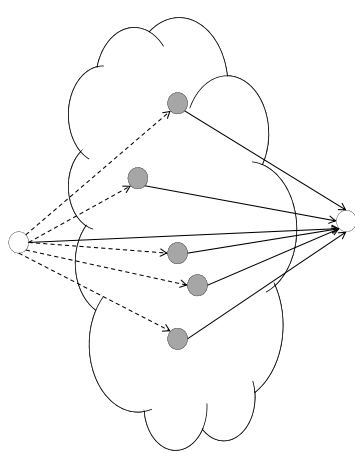

a)

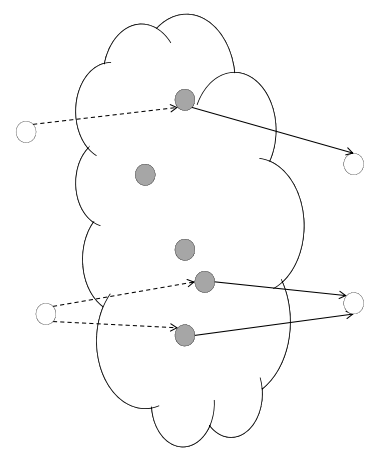

b)

Figure 1: Cooperation and resource reuse trade-off: a) all nodes cooperate for a single transmission, b) partial cooperation with parallel transmissions.

In this paper, we provide an optimization approach to compare the performance of cooperative relaying with that of conventional multi-hop forwarding. To this end, we have adopted the following modeling approach:

- We consider a decode-and-forward mechanism. The model can be extended to the case of amplify-and-forward, although it would lead to a non-linear and discrete optimization problem that is beyond the scope of the current paper.

- We account for interference with the physical model that considers a minimum threshold on the signal-to-interference and noise Ratio (SINR) [26]. With cooperation relaying, we use the sum of the contributing signals in the overall SINR. This allows to evaluate accurately the effect of resource reuse without the limitations of the protocol interference model.

- We capture the channel quality improvement of cooperative relaying in terms of increased transmission range. The model can be extended to a discrete set of multiple transmission rates and SINR thresholds at the cost of a higher complexity. 
- Since both packet routing and transmission scheduling greatly impact the overall network capacity, we jointly optimize them considering a Spacial Time Division Multiple Access (STDMA) approach in order to make the comparison fair. This also allows us to neglect the effect of protocol overheads in the analysis.

- Our model works for arbitrary network topologies and optimizes the path selection and the transmission scheme at each hop with or without cooperation. Therefore, for the cooperation case, the optimization includes the decision of optimal relay selection.

- We assume half-duplex operation of wireless devices that, therefore, cannot transmit and receive at the same time.

It is easy to see that most of the above assumptions define a comparison scenario that is favorable to cooperative relaying, and the conventional multi-hop forwarding scheme is just a special case of our cooperative relaying framework. This allows us to measure the relative gain in network efficiency selecting the best level of cooperation. The cooperation gain we calculate can be considered an upper limit that may not be reachable in practice due to the overhead required by cooperation. We show, however, that even for this upper limit, the cooperation gain may be quite small in some network settings where multiple connections must be served in parallel and the interference level is high.

It is worth pointing out that the optimization framework we present does not define a new cooperation algorithm, and hence the research theme differs from the scope of many practical cooperation algorithms that have been proposed in the literature. The proposed approach extends and generalizes classical optimization schemes for routing and scheduling in non-cooperative networks [3033]. Therefore, it also has a practical value in those network scenarios where propagation and traffic conditions are quite stable and a centralized scheme can be used to optimize radio resources, like for example in wireless mesh networks. To the best of our knowledge, this is the first approach that provides 
a framework for optimization of radio resources (routing, scheduling, and relay selection) in cooperative relaying and that allows a vis-a-vis comparison with conventional multi-hop forwarding.

The paper is organized as follows. Section 2 is devoted to the review of related work. In Section 3 we present the system model and the related assumptions. In Section 4 we propose an integer programming formulation of the cooperation optimization problem, and in Section 5 we present a solution approach based on column generation. Computation results on some illustrative examples and on a larger set of randomly generated networks are provided in Section 6. Finally, Section 7 concludes the paper.

\section{Related Work}

There is a quite large amount of literature on the channel capacity of various cooperative relaying schemes. Research in this area has taken into consideration several aspects including distributed coding schemes [13-15], retransmission mechanisms [16], and power allocation methods $[17,18]$. In addition to capacity analysis, it has been shown that cooperative relaying can provide some spacial diversity gain due to the fading effect of the radio channel caused by obstacles and multi-path propagation $[8,19]$, even though there is an obvious trade-off between capacity and diversity gains [20].

On the other hand, network-level evaluation of the performance gain of cooperative relaying in wireless multi-hop network has received so far little attention in the literature. It amounts to measuring the overall efficiency in using radio resources when serving multiple traffic flows between different source-destination pairs in presence of interference. In fact, due to the difficulty of modeling interference, network capacity evaluation is not an easy task even with the conventional multi-hop forwarding scheme, for which mainly asymptotic results exist $[26]$.

A first work in [27] considers cooperative relaying in a network with two sources and two destinations, and evaluates the channel capacity. Differently 
from [27], our study is characterized by the consideration of arbitrary ad hoc network topologies and the focus on the evaluation of network capacity.

Modeling the interference from a statistical perspective, the authors of [28] consider ad hoc network scenarios with multi-user interference and analyze the behavior of the amplify-and-forward scheme. We consider instead the decodeand-forward scheme and evaluate the network efficiency in using radio resources when simultaneous transmissions can be coordinated and interference-controlled through an optimal scheduling scheme.

In [29], the gain of cooperative relaying in large networks with multiple sources and destinations and amplify-and-forward relays is evaluated, and asymptotic results are provided. Interference is modeled using a conflict graph according to the protocol model. In our work, we address interference with the more accurate physical model based on SINR, and propose a different approach for evaluating cooperative relaying and optimizing resource management, in order to provide a method for defining optimal scheduling, relay selection and routing schemes in arbitrary-topology networks.

\section{System Model}

\subsection{Model overview}

The approach we use to define a mathematical model of the system and to formulate the resource optimization problem uses two types of graphs. Specifically, we define a Physical Topology Graph and a set of Cooperation Graphs with different levels $\kappa$ of cooperation.

The physical topology graph defines the set of possible direct communications between wireless devices (graph nodes) without any cooperative relaying. In the graph, a (direct) link exists if and only if the signal-to-noise ratio (SNR) at the receiving node is above a threshold, and the interference constraint is defined according to the classical physical model [26].

The cooperation graph defines the set of possible direct communications between wireless devices in case of cooperative relaying. In this graph, a node 
corresponds to a set of cooperating devices, and a link defines the possible direct transmission from a set of transmitting devices to a set of receiving devices. The level of cooperation is defined by the maximum cardinality of the set of cooperating devices that may form a node in the cooperation graph. The existence of a link in the cooperation graph depends on the SNR at all the receiving devices, where the signals of all transmitting devices are summed up. The interference constraint is defined by extending the physical model and considering the SINR at all receiving nodes, where the signal contributions from all transmitting nodes are put together. In the following we present the system model in a mathematically formal way.

\subsection{Physical Topology Graph}

The physical topology is represented by a directed graph $\mathcal{G}=(\mathcal{N}, \mathcal{L})$, where $\mathcal{N}$ and $\mathcal{L}$ denote the sets of nodes and communication links, respectively. Denote by $P_{v}$ the transmission power of $v \in \mathcal{N}, g_{v w}$ the radio propagation gain between $v$ and $w$, and $\eta$ the effect of thermal noise. A link $(v, w)$ exists if and only if its SNR is above a threshold $\gamma$ :

$$
S N R_{v w}=\frac{P_{v} g_{v w}}{\eta} \geq \gamma .
$$

With time division multiple access, radio transmissions are organized using time slots, each of which may accommodate one or several parallel transmissions. Transmission between two nodes generates interference at the other receivers. Suppose link $(v, w)$ is used for a transmission from $v$ to $w$, and $K$ is the set of transmitting nodes in addition to $v$ in the same time slot. The transmission on $(v, w)$ is considered successful if and only if the signal-to-interference-and-noise ratio (SINR) is at least $\gamma$, i.e.,

$$
S I N R_{v w}=\frac{P_{v} g_{v w}}{\sum_{u \in K} P_{u} g_{u w}+\eta} \geq \gamma .
$$




\subsection{Performance Metric and Problem Definition}

A widely used performance metric in scheduling transmissions is the number of time slots required to deliver traffic demand. The metric is directly related to the overall efficiency in using radio resources. In comparing cooperative relaying with conventional forwarding, this metric captures the efficiency of resource reuse, i.e., the minimization of the number of slots through parallel transmissions, as well as the effect of range extension by cooperative relaying, which potentially reduces the required number of transmissions.

To this end, we consider a demand set $\mathcal{D}$; each element $d \in \mathcal{D}$ is a packet associated with a tuple $\left\langle o^{d}, t^{d}\right\rangle$, where $o^{d}$ are $t^{d}$ are the source and destination of the packet, respectively. Without cooperative relaying, each transmission involves exactly one sender and one receiver, and takes place over one link in $\mathcal{L}$. With cooperation in transmission, multiple nodes may simultaneously transmit or receive the same packet, and a receiver can combine the signals from multiple senders, which may or may not have links to the receiver in the physical topology $\mathcal{G}$.

Definition 1. A wireless network $\mathcal{G}$ has a $\kappa$-level of cooperation, if, during the same time slot, at most $\kappa$ nodes are allowed to transmit the same packet to a set of one or more receiving nodes, and each receiver can combine the signals from the $\kappa$ transmitters.

Our comparative study of network performance seeks the answer to the following question: What are the minimum numbers of time slots required to deliver the given set of packets with and without cooperation, respectively? This leads to an optimization problem in routing and scheduling the packet set $\mathcal{D}$ in network $\mathcal{G}$ under a $\kappa$-level of cooperation, where a non-cooperative network corresponds to $\kappa=1$.

\subsection{Communication Graph with Cooperation}

For $\kappa>1$, graph $\mathcal{G}$ is not sufficient for modeling cooperative transmission. We develop a graph concept, which we refer to as the $\kappa$-Cooperation graph, to 
generalize the original topology $\mathcal{G}$.

Definition 2. For graph $\mathcal{G}$, the $\kappa$-Cooperation Graph $G_{\mathcal{G}, \kappa}=(V, A)$, is the auxiliary graph representing all possible transmissions in $\mathcal{G}$ permitted by a $\kappa$ level of cooperation. A node $i \in V$ represents a non-empty subset of $\mathcal{N}$ having cardinality up to $\kappa$, and a link $(i, j) \in A$ represents simultaneous transmission of one packet from all nodes in the $i$ 's node set in $\mathcal{N}$ to $j$ 's node set in $\mathcal{N}$.

Henceforth, we simplify the notation of the $\kappa$-cooperation graph to $G$, when there is no ambiguity. For the sake of clarity, we use $v$ and $w$ to denote nodes in the original graph $\mathcal{G}$, and $i$ and $j$ nodes in the cooperation graph $G$. Nodes and links in $G$ are also referred to as super-nodes and super-links. Let $\Gamma(i)$ denote the set of nodes in $\mathcal{N}$ forming super-node $i$ in $V$, and $\Lambda(i, j)$ the set of links forming super-link $(i, j) \in A: \Lambda(i, j)=\{(v, w) \mid v \in \Gamma(i), w \in \Gamma(j)\}$. Note that the size of $G$ grows exponentially in $\kappa$. When $\kappa=1$, the $\kappa$-cooperation graph $G$ reduces to the original topology $\mathcal{G}$. The concepts of super-node and super-link are illustrated in Figure 2. In this example, each of the two super-nodes $i$ and $j$ contains two nodes in $\mathcal{N}$, and the super-link $(i, j)$ represents transmissions from all nodes in $\Gamma(i)$ to all nodes in $\Gamma(j)$.

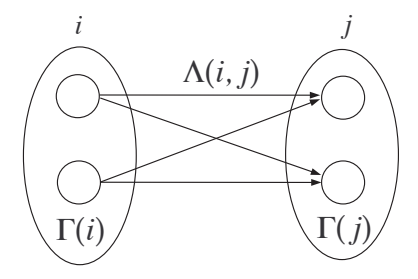

Figure 2: An illustration of super-nodes and super-link.

In Figure 2, the four transmissions do not necessarily correspond to links in the original graph $\mathcal{G}$. This is because the SNR condition takes a new form in the cooperation graph $G$. For a super-link $(i, j) \in A$ to exist, the following SNR condition applies to all the receivers of the super-link, i.e., for all $w \in \Gamma(j)$ :

$$
S N R_{i w}=\frac{\sum_{v \in \Gamma(i)} P_{v} g_{v w}}{\eta} \geq \gamma
$$


The numerator in (3) models the fact that the nodes in $\Gamma(i)$ are transmitting the same packet and hence all contributing to improving SNR. For this reason, a super-link can be established, as a result of cooperation, even if some or possibly none of the transmissions of this super-link is part of the link set in the original topology.

When several super-nodes are transmitting in the same time slot, interference must be accounted for. Suppose in addition to $i$, a set of super-nodes $\Omega$ are transmitting in the same time slot. The SINR condition for super-link $(i, j)$ is that the following inequality holds for all $w \in \Gamma(j)$ :

$$
S I N R_{i w}=\frac{\sum_{v \in \Gamma(i)} P_{v} g_{v w}}{\sum_{l \in \Omega} \sum_{u \in \Gamma(l)} P_{u} g_{u w}+\eta} \geq \gamma
$$

In (4), all nodes composing the super-nodes in $\Omega$ generate interference to super-link $(i, j)$. Note that a node $u \in \mathcal{N}$ may appear at most once in the denominator, because the super-nodes transmitting in any time slot all must have mutually disjoint sets of nodes in the original graph.

\subsection{Classes of Super-Links}

The super-nodes in $G$ vary in the cardinality of the associated subsets of nodes in the original graph. Based on this cardinality, we define four classes of super-links.

1. One-to-one. These are the links in the original physical topology, i.e., links in $\mathcal{L}$. Super-link $(i, j)$ is of this class if it satisfies the condition: $\Gamma(i)=\{v\}, \Gamma(j)=\{w\}$, and $(v, w) \in \mathcal{L}$.

2. One-to-many. A super-link of this class corresponds to a group of links in $\mathcal{L}$ originating from the same node in $\mathcal{N}$. Thus $(i, j) \in A$ is a one-tomany super-link if $\Gamma(i)=\{v\},|\Gamma(j)|>1$, and $(v, w) \in \mathcal{L}$ for all $w \in \Gamma(j)$. The one-to-many links are also referred to as broadcast links. A special subclass of one-to-many links is the so called buffering links, in which a node behaves as if it is transmitting also to itself. 
3. Many-to-one. A super-link $(i, j) \in A$ is of class many-to-one, if $|\Gamma(i)|>1$ and $\Gamma(j)=\{w\}, w \in \mathcal{N} \backslash \Gamma(i)$. This super-link represents simultaneous transmissions of the same packet from all nodes in $\Gamma(i)$ to the single receiver $w$ in $\Gamma(j)$. A many-to-one link does not necessarily consist in a group of links in the original graph, i.e., $\Gamma(i)$ may contain node $v$ for which $(v, w) \notin \mathcal{L}$, provided that the SNR at $w$ satisfies (3) by cooperation. Links in the many-to-one class are also referred to as cooperating links.

4. Many-to-many: This class of links in $G$ represents transmissions of the same packet between multiple transmitters and receivers in the original graph $\mathcal{G}$. A super-link $(i, j)$ is a many-to-many link if and only if $|\Gamma(i)|>1$ and $|\Gamma(j)|>1$. The super-link shown in Figure 2 is of this class. Similar to many-to-one super-links, a many-to-many super-link $(i, j)$ can be created by cooperation, hence it may have transmissions between one or multiple pairs of nodes $v \in \Gamma(i)$ and $w \in \Gamma(j)$ for which $(v, w) \notin \mathcal{L}$. Many-to-many super-links are also called broad-cooperating links or multicasting links.

Example 1. Figure 3 shows the physical topology of a wireless network with 5 nodes. Figure 4 shows the super-nodes of the corresponding 2-cooperation network. For clarity, only a few of the super-links are drawn. The dot-dashed links are of class one-to-one; these are the links incident to node 2 in the original topology. The solid super-links are broadcasting links from node 3 to super-nodes $\{1,2\},\{1,5\}$, and $\{2,5\}$, respectively. Each of the three dotted super-links is a cooperating link, representing simultaneous transmissions of a packet from super-node $\{2,3\}$ to a single receiver. Note that transmissions on $(2,1),(2,5)$, and $(3,4)$ do not correspond to any physical link in Figure 3. Finally, the dashed super-links are multicasting links, each of which carries transmissions involving two transmitters and two receivers; some of these transmissions occur between nodes that do not have links in Figure 3. The distance in hops between a source-destination pair may benefit from cooperating and multicasting links. For example, the shortest path from node 4 to node 1 has three hops if no cooperation 


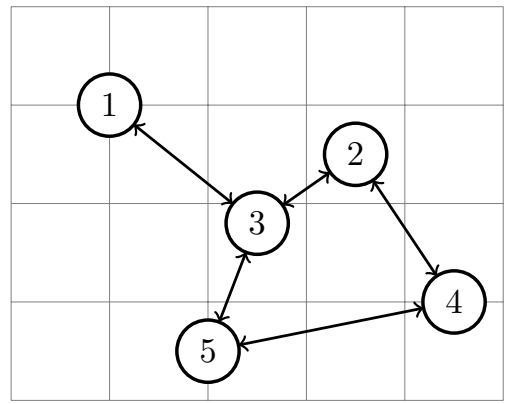

Figure 3: Topology of a very simple wireless network.

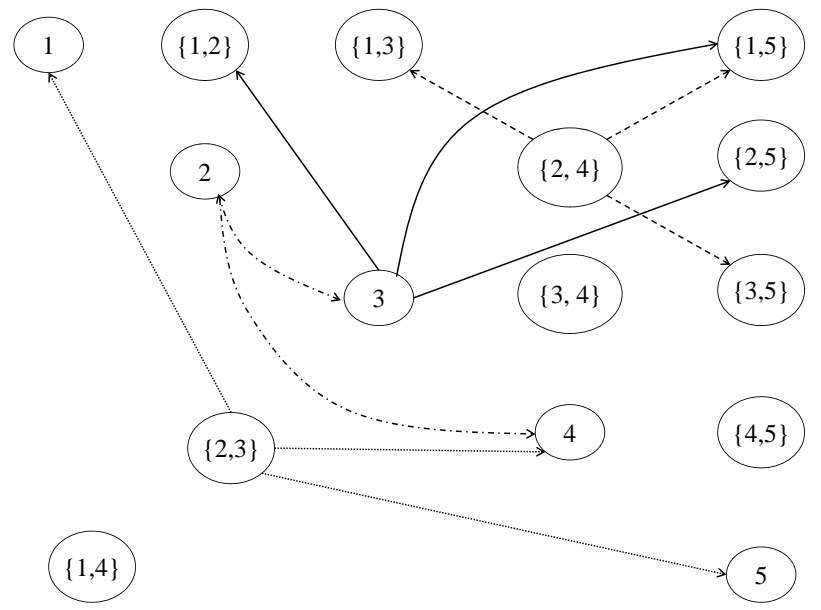

Figure 4: Super-nodes and some super-links of the 2-cooperation graph for the network of Figure 3.

is allowed. The shortest path distance in the cooperation graph becomes two hops, and one of such paths is formed by $4,\{2,5\}$, and 1 .

\section{An Integer Programming Formulation}

Routing and scheduling of packets without cooperation, with the objective of minimizing the total number of time slots, has been studied quite extensively in the literature (see Section 1). From a computational complexity standpoint, this optimization problem is $N P$-hard, in particular because even if optimization 
is restricted to scheduling only (i.e., routing is fixed), the problem generalizes graph coloring [30].

It has been shown that integer programming formulations based on identifying feasible configurations admits, within a reasonable amount of computing time, an optimal or very-close-to optimal routing and scheduling solution for networks of up to moderate size [32, 33].

The key element in these formulations is the definition of a feasible configuration that refers to a group of transmissions that may share a time slot (simultaneous transmissions) without violating the SINR constraint at any receiver. Each feasible configuration is associated with a 0-1 decision variable, and the problem formulation defines which configurations to activate in different time slots in order to minimize their total number.

Utilizing the cooperation graph, we are able to generalize this integer programming concept to networks with cooperation. For cooperation graph $G$, a feasible configuration of a time slot is a group of super-links, such that the SINR condition (4) is satisfied for all of them under simultaneous transmissions. Thus constructing a scheduling solution amounts to selecting a set of feasible configurations, and the cardinality of the set equals the total number of time slots.

Let $\mathcal{S}$ denote the collection of all feasible configurations, and $S_{i j} \subseteq \mathcal{S}$ the set of configurations containing super-link $(i, j) \in A$. Let $b_{i}^{d}$ be a parameter that is equal to 1 if node $i \in \mathcal{N} \cap V$ is the source of the packet $d,-1$ if it is the destination of $d$, and 0 otherwise. We define two sets of optimization variables.

$$
\begin{aligned}
& \lambda_{s}=\text { The number of time slots allocated to configuration } s \in \mathcal{S}, \\
& \beta_{i j}^{d}= \begin{cases}1 & \text { if super-link }(i, j) \text { is used to route packet } d \text { from } i \text { to } j, \\
0 & \text { otherwise. }\end{cases}
\end{aligned}
$$

The problem of routing and scheduling the demand set $\mathcal{D}$ with a minimum number of time slots can be formulated as the following integer linear programming model. 


$$
\begin{array}{ll}
{[\mathbf{P}]} & \min \sum_{s \in \mathcal{S}} \lambda_{s} \\
\text { s.t. } & \sum_{(i, j) \in A} \beta_{i j}^{d}-\sum_{(j, i) \in A} \beta_{j i}^{d}=b_{i}^{d}, i \in V, d \in \mathcal{D}, \\
& \sum_{s \in S_{i j}} \lambda_{s} \geq \sum_{d \in \mathcal{D}} \beta_{i j}^{d},(i, j) \in A, \\
& \beta_{i j}^{d} \in\{0,1\},(i, j) \in A, d \in \mathcal{D}, \\
& \lambda_{s} \in \mathbb{Z}^{+}, s \in \mathcal{S} .
\end{array}
$$

The objective is to minimize the total number of configurations (i.e., time slots). Constraints (6) are the classical flow balance equations for each packet $d \in \mathcal{D}$. They define the routing from the sources to the destinations, and incorporate the decision of relay selection in the cooperative scheme. Constraints (7) link the flow variables $\beta$ to the configuration variables $\lambda$ : For each super-link

$(i, j) \in A$, sufficiently many configurations containing $(i, j)$ must be chosen to accommodate the total flow on $(i, j)$.

\section{A Column Generation Approach}

\subsection{The Framework}

The number of $\lambda$-variables in $\mathbf{P}$ equals $|\mathcal{S}|$, which is exponential in the size of the cooperation graph $G$. Thus a solution algorithm requiring the generation of all possible feasible configurations a priori is not computationally feasible. Exploiting the structure of $\mathbf{P}$, an effective solution strategy is to solve its linear programming (LP) relaxation by means of a column generation method, which provides a lower bound on the optimal number of time slots, followed by computing the optimal or a near-optimal integer solution (see, e.g., [34] for a textbook on column generation). By keeping a small subset $\mathcal{S}^{\prime} \subset \mathcal{S}$ and expanding the subset systematically, the column generation method decomposes the LP version of $\mathbf{P}$ into a master problem and a subproblem. The former is used to find the optimal solution when the optimization is restricted to the elements in 
$\mathcal{S}^{\prime}$. The latter corresponds to a separation problem for the dual LP for checking optimality, that is, to either find additional elements that are of interest to be added to $\mathcal{S}^{\prime}$, or conclude that the current solution of the master problem is optimal not only for $\mathcal{S}^{\prime}$ but also for $\mathcal{S}$ (i.e., all variables $\lambda_{s}$ for $s \in \mathcal{S} \backslash \mathcal{S}^{\prime}$ are zeros at optimum).

The master problem looks very similar to $\mathbf{P}$. To save space, we do not present it in mathematical form. The master problem differs from $\mathbf{P}$ in the following two aspects.

1. Set $\mathcal{S}$ is replaced by a subset $\mathcal{S}^{\prime}$, of which $\left|\mathcal{S}^{\prime}\right| \ll|\mathcal{S}|$.

2. The integrality requirements on the $\beta$ - and $\lambda$-variables are relaxed.

As long as the resulting master problem contains at least one feasible solution, any $\mathcal{S}^{\prime} \subset \mathcal{S}$ can serve as the starting point. That is, the subset $\mathcal{S}^{\prime}$ must be such that constraints (7) can be satisfied. The simplest choice consists of setting $\mathcal{S}^{\prime}=A$, that is for each super-link $(i, j)$ in $A$ we have a configuration where $(i, j)$ is the only active link.

\subsection{The Pricing Problem}

After solving the master problem, checking the LP optimality condition amounts to examining whether or not any $\lambda_{s}, s \in \mathcal{S} \backslash \mathcal{S}^{\prime}$, has a negative reduced cost. Denoting the dual variables of $(7)$ by $\bar{\pi}_{i j},(i, j) \in A$, the reduced cost of $\lambda_{s}$ equals $1-\sum_{(i, j) \in A: s \in S_{i j}} \bar{\pi}_{i j}$. The subproblem, also called the pricing problem, takes the following form,

$$
\min _{s \in \mathcal{S} \backslash \mathcal{S}^{\prime}}\left(1-\sum_{(i, j) \in A: s \in S_{i j}} \bar{\pi}_{i j}\right) .
$$

The minimization in (10), in its turn, can be formulated by an integer linear model; thus we can solve (10) without explicitly having the set $\mathcal{S}$. A solution of this integer model corresponds to a feasible configuration, that is, a set of super-links that can be active in the same time slot. Below we define the set of binary variables used by the model, and the model itself. 


$$
\begin{aligned}
& z_{i j}= \begin{cases}1 & \text { if super-link }(i, j) \text { is active } \\
0 & \text { otherwise }\end{cases} \\
& \text { [R1] } \min -\sum_{(i, j) \in A} \bar{\pi}_{i j} z_{i j} \\
& \text { s.t. } \sum_{\substack{(i, j) \in A: \\
v \in \Gamma(i)}} z_{i j}+\sum_{\substack{(j, i) \in A: \\
v \in \Gamma(i)}} z_{j i} \leq 1, \quad v \in \mathcal{N} \\
& \frac{\sum_{v \in \Gamma(i)} P_{v} g_{v w}}{\sum_{\substack{k, l) \in A: \\
k \neq i, l \neq j}} \sum_{u \in \Gamma(k)} P_{u} g_{u w} z_{k l}+\eta} \geq \gamma z_{i j}, \quad(i, j) \in A, w \in \Gamma(j), \\
& z_{i j} \in\{0,1\}, \quad(i, j) \in A .
\end{aligned}
$$

The objective is to find a configuration of minimum reduced cost. Constraints (12) ensure that the $z$-variables satisfy two conditions. First, for any node $v$ in the original topology $\mathcal{G}$, at most one super-link, of which the starting super-node contains $v$, can be active. In effect, $v$ is allowed to transmit at most one packet in a time slot with or without cooperation. Second, if node $v$ is transmitting, it can not be a receiving node of any active super-link at the same time. The SINR conditions are formulated by (13). The constraint has effect only if $z_{i j}=1$. In this case, the inequality states that the SINR, in which the interference originates from all active super-links other than $(i, j)$, must be at least $\gamma$. Note that for any super-node $k, k \neq i$, the outer-sum in the denominator will contain at most one super-link from $k$, because of (12).

Constraints (13) are non-linear. A standard way of linearizing these constraints (e.g., [35]) is to introduce a parameter, denote by $M$, and reformulate (13) by the following linear inequalities. The parameter $M$ is chosen to be large enough such that the inequality has no effect if $z_{i j}=0$.

$$
\sum_{v \in \Gamma(i)} P_{v} g_{v w} \geq \gamma\left(\sum_{\substack{k, l) \in A: \\ k \neq i, l \neq j}} \sum_{u \in \Gamma(k)} P_{u} g_{u w} z_{k l}+\eta\right)-M\left(1-z_{i j}\right),(i, j) \in A, w \in \Gamma(j) .
$$


Computationally, the number of SINR constraints (15) has a great impact on the time required to solve the pricing problem. Recall that the number of super-links $|A|$ is exponential in the size of the original graph $\mathcal{G}$. In $\mathbf{R} 1$, there are $\sum_{(i, j) \in A}|\Gamma(j)|$ SINR constraints. Below we present an alternative model for the pricing problem using more variables and inequalities but significantly less SINR constraints.

$$
\begin{aligned}
& q_{v w}= \begin{cases}1 & \text { if node } v \in \mathcal{N} \text { is transmitting to } w \in \mathcal{N} \\
0 & \text { otherwise }\end{cases} \\
& q_{v w}^{\prime}= \begin{cases}1 & \text { if transmission of node } v \in \mathcal{N} \text { gives interference at } w \in \mathcal{N} \\
0 & \text { otherwise }\end{cases} \\
& x_{v}= \begin{cases}1 & \text { if node } v \in \mathcal{N} \text { is transmitting } \\
0 & \text { otherwise } \\
1 & \text { if node } w \in \mathcal{N} \text { is receiving } \\
0 & \text { otherwise }\end{cases} \\
& {[\mathbf{R 2}] \min -\sum_{(i, j) \in A} \bar{\pi}_{i j} z_{i j}}
\end{aligned}
$$$$
\text { s.t. }(12) \text {, }
$$

$$
\begin{array}{lr}
\sum_{\substack{(i, j) \in A \\
v \in \Gamma(i), w \in \Gamma(j)}} z_{i j}=q_{v w}, & v \in \mathcal{N}, w \in \mathcal{N}, v \neq w, \\
x_{v}=q_{v w}+q_{v w}^{\prime}, & v \in \mathcal{N}, w \in \mathcal{N}, v \neq w, \\
q_{v w} \leq y_{w}, & v \in \mathcal{N}, w \in \mathcal{N}, v \neq w, \\
\frac{\sum_{v \in \mathcal{N}} P_{v} g_{v w} q_{v w}}{\sum_{u \in \mathcal{N}} P_{u} g_{u w} q_{u w}^{\prime}+\eta} \geq \gamma y_{w}, & w \in \mathcal{N}, \\
z_{i j} \in\{0,1\}, & (i, j) \in A, \\
q_{v w}, q_{v w}^{\prime} \in\{0,1\}, & v \in \mathcal{N}, w \in \mathcal{N}, v \neq w, \\
x_{v}, y_{v} \in\{0,1\}, & v \in \mathcal{N} .
\end{array}
$$

By (16), node $v$ transmits to node $w$ if and only if a super-link containing $v$ and $w$ as transmitting and receiving nodes, respectively, is active. Constraints 
(17) state that, if $v$ is transmitting, then another node $w$ either is a receiver, or experiences interference from $v$. The next set of constraints ensures $y_{w}=1$ if $q_{v w}=1$ for any $v$. The SINR conditions, one for each $w \in \mathcal{N}$, are formulated in (19). These constraints can be linearized in a way similar to (15).

\subsection{Algorithm Summary}

The computational machinery that we propose for solving the routing and scheduling problem in a cooperative network consists in the following steps.

Step 1. Define an initial subset $\mathcal{S}^{\prime} \subset \mathcal{S}$.

Step 2. Solve the master problem of the LP relaxation of $\mathbf{P}$ (Section 5.1).

Step 3. Solve the pricing problem using either of the two models (Section 5.2).

Step 4. If a configuration $s \in \mathcal{S} \backslash \mathcal{S}^{\prime}$ with negative reduced cost is found, add $s$ to $\mathcal{S}^{\prime}$ and go to Step 2, otherwise go to the next step.

Step 5. Find an integer solution for the configurations in set $\mathcal{S}^{\prime}$.

The last step can be performed by applying an integer linear solver, or, if this takes excessive computing time, a heuristic algorithm. In either case, $\mathbf{P}$ is not guaranteed to be solved to global optimality, because some configurations used at integer optimum may not be present in $\mathcal{S}^{\prime}$ in this step. Ensuring integer optimality would require embedding the above algorithm into a branchand-price scheme. Empirically, however, for all the network instances used in our experiments, integer optimum is obtained and verified (by the LP value, sometimes after rounding), i.e., no branching is needed.

Among the steps in the algorithm, two are crucial to computational efficiency. The first is the initial subset of configuration $\mathcal{S}^{\prime}$, and the second is the solution of the pricing problem. In order to generate $\mathcal{S}^{\prime}$, we use a Shortest Path Allocation (SPA) heuristic that generates, for each demand $d \in \mathcal{D}$, the shortest path from its source $o^{d}$ to its destination $t^{d}$, and adds to $\mathcal{S}^{\prime}$ one configuration per link that appears in at least one of the paths. The step of solving the pricing subproblem is definitely the computational bottleneck. One approach to speed up this step is not to solve the pricing problem to optimality (i.e., finding the 
minimum reduced cost), but to terminate once a configuration with negative reduced cost is found; this configuration is added to the master problem. Although the number of column generation iterations grows, the approach pays off as significantly less time is spent per iteration.

\section{Computational Results}

We present first a set of illustrative examples to highlight some insights into the performance gain of cooperative relaying. Then, in the second subsection, we report the bulk of computational results on a large set of random instances.

\subsection{Illustrative Examples}

A clear advantage of cooperative relaying is to enable communication between nodes that, due to the SNR constraint, are not connected in the original network. Figure 5 shows a small example of a network with 6 nodes and two demands $\langle 2,4\rangle$ and $\langle 4,3\rangle$. Recall that, in this paper, a demand represents a single packet associated with a source and a destination. The transmission power $P=0.2 \mathrm{~mW}$, the thermal noise at the receiver $\eta=10^{-10} \mathrm{~mW}$, and the SNR threshold $\gamma=10$. Since node 4 is not connected to node 2 or node 3 , the two demands cannot be satisfied without cooperation. If we allow a 2-level of cooperation, the demand $\langle 2,4\rangle$ is satisfied with the configurations $2 \rightarrow\{3,5\}$ and $\{3,5\} \rightarrow 4$, and the demand $\langle 4,3\rangle$ with the configurations $4 \rightarrow\{4,6\}$ and $\{4,6\} \rightarrow 3$. Note that the second demand uses the buffering link $4 \rightarrow\{4,6\}$.

The advantage of cooperation tends to decrease as the connectivity of the network increases. To quantify the connectivity, we use two network properties: (i) the network density $\delta=\frac{\mathcal{L}}{\mathcal{N}(\mathcal{N}-1)}$, that is the percentage of links that satisfy the SNR constraint with respect to a fully connected network, and (ii) the network diameter $\Delta$, that is, the maximum hop distance between any pair of nodes. Figures 6 and 7 show two networks that differ only in the level of connectivity. In the first case the nodes transmit with $P=0.1 \mathrm{~mW}$. In the second case the power $P=0.4 \mathrm{~mW}$. In both cases, each pair of nodes $i$ and 


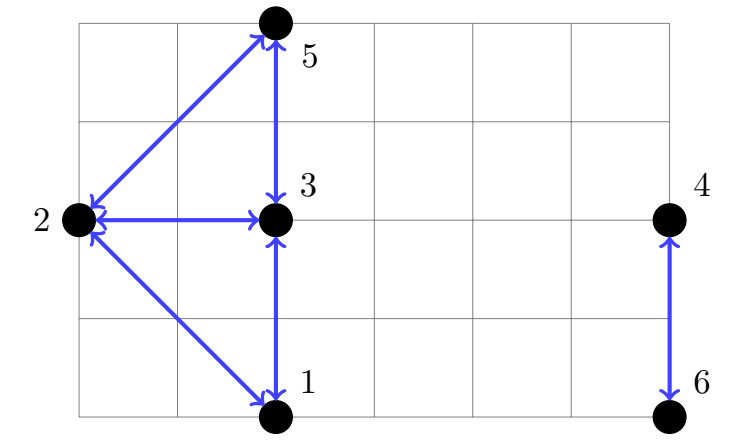

(a) Topology with $P=0.2 \mathrm{~mW}, \eta=10^{-10} \mathrm{~mW}, \gamma=$ 10 .

\begin{tabular}{|l|l|l|l|}
\hline $2 \rightarrow 3,5$ & $3,5 \rightarrow 4$ & $4,6 \rightarrow 3$ & $4 \rightarrow 4,6$ \\
\hline
\end{tabular}

(b) Routing and scheduling two demands $\langle 2,4\rangle$ and $\langle 4,3\rangle$

Figure 5: Cooperative networking to provide additional connectivity.

$j$ has a demand in both directions, that is $\langle i, j\rangle$ and $\langle j, i\rangle$. For the network in Figure 6 , the optimal solution requires 130 time slots without cooperation, and only 110 time slots with cooperation, showing a clear advantage of cooperation. In contrast, for the network in Figure 7 where the connectivity is much higher, the optimal schedule length is 74 , with or without cooperation.

The traffic load, measured in the number of traffic demands, is another important factor influencing the potential of cooperation. In particular, increasing the number of traffic demands, the advantage of cooperative relaying decreases. Figure 8 shows an illustrative example of a grid network with 12 nodes, $P=0.2$ $\mathrm{mW}, \eta=10^{-10} \mathrm{~mW}$, and $\gamma=10$. We compare two problem instances: the first instance has a single demand $\langle 1,4\rangle$, the second instance has two demands $\langle 1,4\rangle$ and $\langle 9,12\rangle$. In the first case, cooperative relaying requires one time slot less to satisfy the demand. In the second case, cooperative networking has an optimal solution where cooperation does not occur, i.e., the optimum is identical to that of conventional hop-by-hop forwarding. 


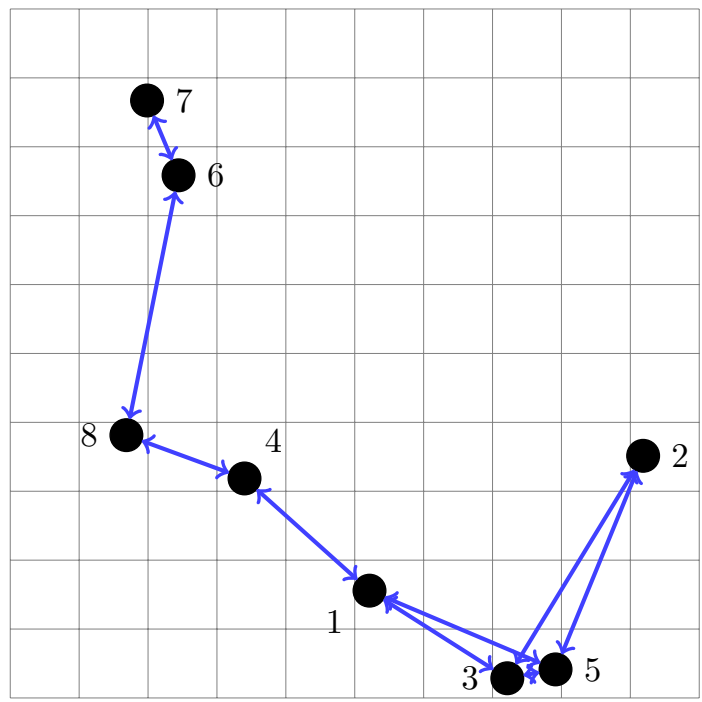

Figure 6: Topology with density $\delta=0.14$ and diameter $\Delta=6$, induced by parameters $P=0.1$ $\mathrm{mW}, \eta=10^{-10} \mathrm{~mW}$, and $\gamma=15$.

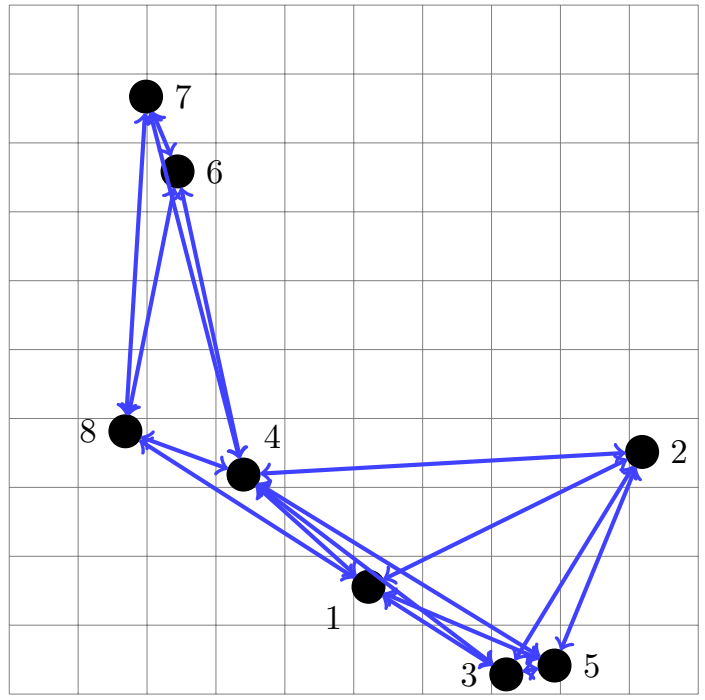

Figure 7: Topology with density $\delta=0.30$ and diameter $\Delta=2$, induced by parameters $P=0.4$ $\mathrm{mW}, \eta=10^{-10} \mathrm{~mW}$, and $\gamma=15$. 


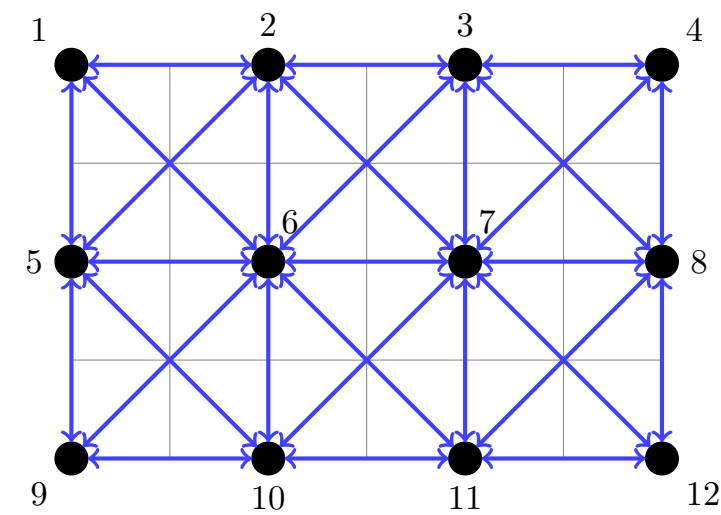

(a) Topology with $P=0.2 \mathrm{~mW}, \eta=10^{-10} \mathrm{~mW}$, and $\gamma=10$.

$$
\begin{array}{|l|l|l|}
\hline 1 \rightarrow 2 & 2 \rightarrow 3 & 3 \rightarrow 4 \\
\hline
\end{array}
$$

(b) Single demand $\langle 1,4\rangle$ : solution without cooperation

$$
\begin{array}{|l|l|}
\hline 1 \rightarrow\{2,6\} & \{2,6\} \rightarrow 4 \\
\hline
\end{array}
$$

(c) Single demand $\langle 1,4\rangle$ : solution with cooperation

$$
\begin{array}{|c|c|c|}
\hline 2 \rightarrow 3 & 3 \rightarrow 4 & 1 \rightarrow 2 \\
10 \rightarrow 11 & 11 \rightarrow 12 & 9 \rightarrow 10 \\
\hline
\end{array}
$$

(d) Two demands $\langle 1,4\rangle$ and $\langle 9,12\rangle$ : no cooperation in the optimal solution

Figure 8: The effect of traffic demand on cooperative networking. 


\subsection{Computational Evaluation}

We have performed an assessment of the proposed models by comparing the efficiency of cooperative relaying to that of hop-by-hop forwarding on random network topologies with up to 15 nodes. A first set of results considers networks with 13 nodes drawn on a square area of $100 \mathrm{~m}^{2}$. The thermal noise is set to $\eta=10^{-10} \mathrm{~mW}$ and the SNR threshold $\gamma=10$. The propagation gain is computed as $g_{i j}=\left(\text { distance }_{i j}\right)^{-3}$. The number of demands ranges from 3 (low traffic load) to 90 (high traffic load), while the transmission power is either $P=0.1 \mathrm{~mW}$ or $P=0.4 \mathrm{~mW}$. For $P=0.1 \mathrm{~mW}$, the SNR requirement results in sparse networks with $\Delta=3$ in average, while for $P=0.4 \mathrm{~mW}$ the networks are denser with $\Delta \leq 2$ in average.

Figures 9 and 10 report the cooperation gain averaged over 10 random instances. Cooperation gain is computed as the percentage difference between the optimal schedule lengths obtained with and without cooperation. Figure 9 reports the cooperation gain in respect of the transmission power with a full demand matrix. Figure 10 shows the performance figure in the number of traffic demands with a fixed transmission power $P=0.1 \mathrm{~mW}$. The results confirm the observations made for the illustrative examples in the previous section.

In particular, Figure 9 shows that the cooperation gain decreases as network connectivity increases. Indeed, as explained in Section 6.1, when the transmission power increases, the connectivity of the network also grows, since more node pairs get their SNRs above the threshold. However, the interference level increases as well, making cooperation less advantageous since it activates more simultaneous transmitters. Moreover, the values of the cooperation gain that are basically upper limits of what can be achieved in practice, are rather small and goes from $6.1 \%$ to $9.9 \%$.

Figure 10 confirms that the cooperation gain decreases as the number of traffic demands increases. The main reason for this behavior is again the interference level that goes up with multiple parallel traffic relations. Moreover, the half-duplex operation prevents nodes from being involved simultaneously in different cooperation groups, and thus reduces the advantage of cooperation 


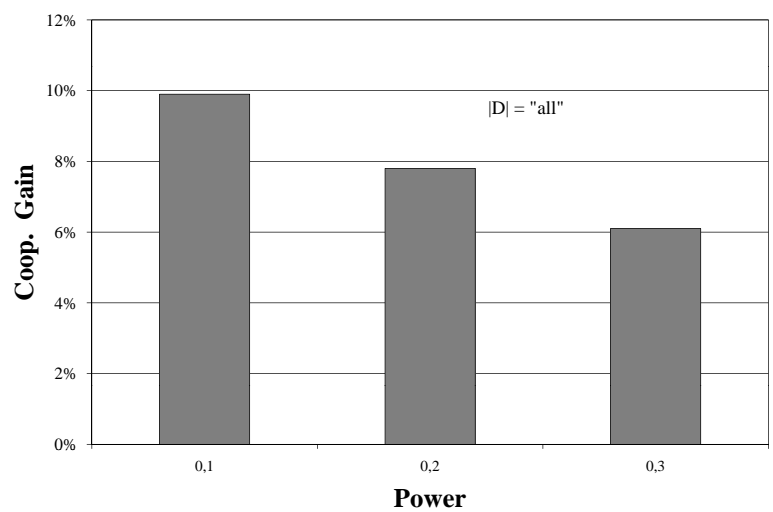

Figure 9: Cooperation gain versus transmission power with full traffic demand matrix.

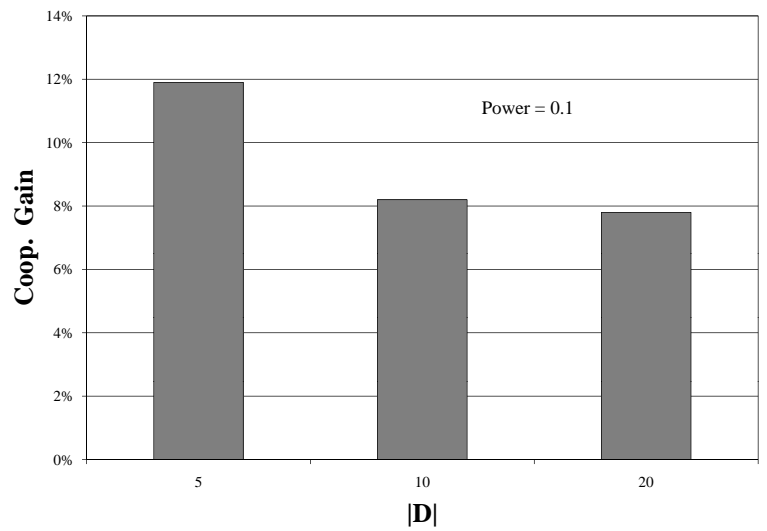

Figure 10: Cooperation gain versus number of traffic demands with fixed transmission power.

with multiple parallel transmissions of different packets. Also, the performance gain is pretty small in value, and ranges between $7.8 \%$ and $11.8 \%$.

Table 1 gives additional details on the same set of instances. For each instance we report the number of links $|\mathcal{L}|$, the number of super-links $|A|$, and the computation time in seconds. Note that, as expected, the computation time is much higher in the case of cooperation, and increases with both the number of demands and the number of links (and thus super-links).

For 10 randomly generated instances, Figures 11, 12 and 13 show results for three power levels, $P=0.1 \mathrm{~mW}, P=0.2 \mathrm{~mW}$, and $=0.3 \mathrm{~mW}$. The instances have 13 nodes and a full demand matrix. The resulting three groups of network 


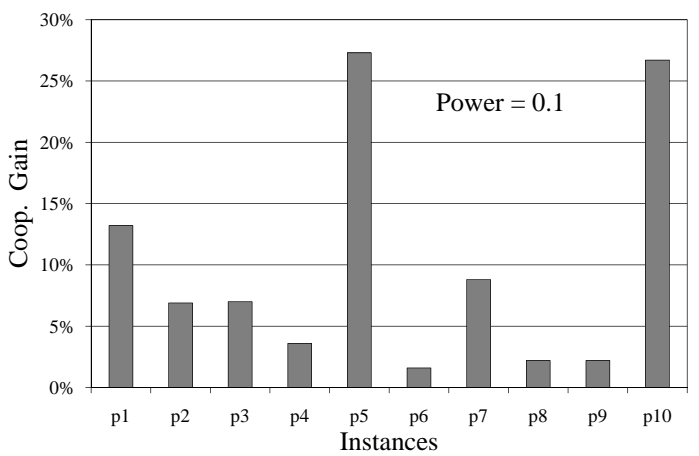

Figure 11: Cooperation gain for 10 random instances with transmission power $P=0.1 \mathrm{~mW}$.

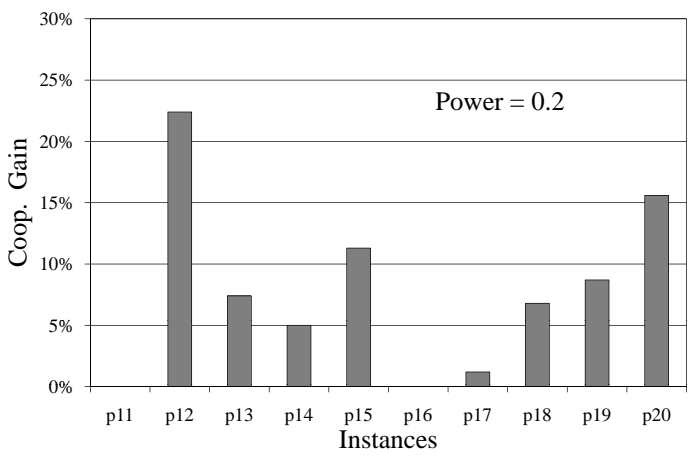

Figure 12: Cooperation gain for 10 random instances with transmission power $P=0.2 \mathrm{~mW}$.

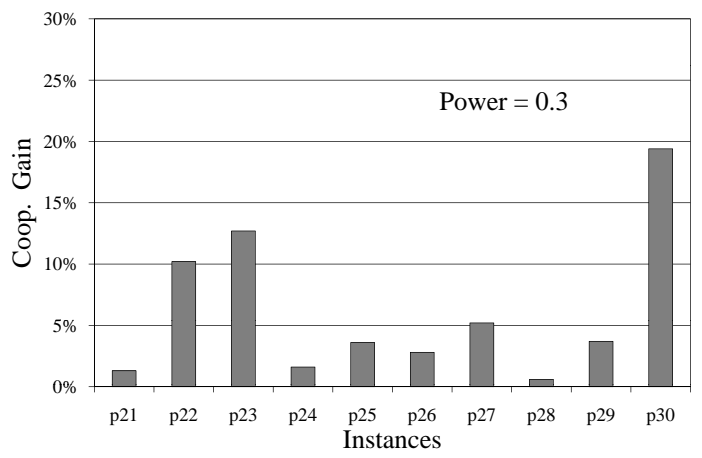

Figure 13: Cooperation gain for 10 random instances with transmission power $P=0.3 \mathrm{~mW}$. 


\begin{tabular}{|c|c|c|c|c|c|c|}
\hline \multirow[b]{2}{*}{ Power } & \multirow[b]{2}{*}{$|D|$} & \multicolumn{2}{|c|}{ No-COOPERATION } & \multicolumn{2}{|c|}{ COOPERATION } & \multirow[b]{2}{*}{ Gain } \\
\hline & & $|\mathcal{L}|$ & Time & $|A|$ & Time & \\
\hline 0.1 & 5 & 42.4 & 0.9 & 626.0 & 16.6 & $11.9 \%$ \\
\hline 0.1 & 10 & 47.2 & 2.2 & 683.2 & 123.0 & $8.2 \%$ \\
\hline 0.1 & 20 & 41.6 & 2.5 & 609.6 & 147.4 & $7.8 \%$ \\
\hline 0.1 & "all" & 41.6 & 5.3 & 609.6 & 194.7 & $9.9 \%$ \\
\hline 0.2 & "all" & 60.8 & 17.8 & 958.6 & 1792.7 & $7.8 \%$ \\
\hline 0.3 & "all" & 76.4 & 23.1 & 1277.6 & 5781.8 & $6.1 \%$ \\
\hline
\end{tabular}

topologies are denoted by p1-p10, p11-p20, and p21-p30, respectively. For each scenario, the corresponding figure reports the cooperation gain. These figures lead to another insight into the behavior of cooperative relaying, namely the very large diversity in the performance gain. Indeed, very different results have been obtained with networks that share the same system parameters. For the three power levels, the ranges of the cooperation gain are [1.6\%,27.3\%], [0\%,22.4\%], and $[0.6 \%, 19.4 \%]$, respectively.

Tables 2, 3 and 4 report detailed results for the instances in Figures 11, 12 and 13. For each instance, the table shows the number of links $\mathcal{L}$, the number of super-links $|A|$, the number of iterations of column generation, and the computation time in seconds. We denote by $L B_{1}$ and $U B_{1}$ the lower and upper bound obtained without cooperation, and $L B_{2}$ and $U B_{2}$ in case of cooperation. The lower bounds are the LP optimum values, and the upper bounds correspond to integer schedule lengths. The last column gives the cooperation gain, computed as $\frac{U B_{2}-U B_{1}}{U B_{2}} * 100$.

Note that our column generation approach produces the global optimal integer solution whenever the ceiling of the lower bound is equal to the upper bound. Inspecting the tables, we observe that this is indeed the case for all the instances.

Finally, Figure 14 provides further insights into scenarios where the advan- 
Table 2: Performance evaluation of random topologies with 13 nodes in a square area of 1000 $m^{2}$, with parameters $P=0.1 \mathrm{~mW}, \gamma=10$, and $\eta=2 * 10^{-10} \mathrm{~mW}$. The time is in seconds.

\begin{tabular}{|c|c|c|c|c|c|c|c|c|c|c|c|}
\hline \multirow[b]{2}{*}{ Ins. } & \multicolumn{5}{|c|}{ No CoOperation } & \multicolumn{5}{|c|}{ COOPERATION } & \multirow[b]{2}{*}{ Gain } \\
\hline & $|\mathcal{L}|$ & Iter & Time & $L B_{1}$ & $U B_{1}$ & $|A|$ & Iter & Time & $L B_{2}$ & $U B_{2}$ & \\
\hline p1 & 38 & 20 & 3.3 & 43.0 & 43 & 525 & 369 & 29.4 & 38.0 & 38 & $13.2 \%$ \\
\hline p2 & 38 & 27 & 6.3 & 324.3 & 325 & 526 & 609 & 309.5 & 303.8 & 304 & $6.9 \%$ \\
\hline p3 & 36 & 22 & 3.3 & 61.0 & 61 & 520 & 190 & 20.6 & 57.0 & 57 & $7.0 \%$ \\
\hline $\mathrm{p} 4$ & 42 & 26 & 5.7 & 115.7 & 116 & 593 & 329 & 151.0 & 112.0 & 112 & $3.6 \%$ \\
\hline p5 & 34 & 13 & 2.8 & 168.0 & 168 & 509 & 316 & 43.0 & 132.0 & 132 & $27.3 \%$ \\
\hline p6 & 34 & 21 & 3.5 & 125.0 & 125 & 510 & 127 & 42.9 & 122.5 & 123 & $1.6 \%$ \\
\hline p7 & 60 & 32 & 14.6 & 236.0 & 236 & 876 & 447 & 1093.4 & 216.5 & 217 & $8.8 \%$ \\
\hline p8 & 38 & 28 & 5.0 & 138.0 & 138 & 581 & 433 & 139.5 & 134.7 & 135 & $2.2 \%$ \\
\hline p9 & 50 & 8 & 3.3 & 95.0 & 95 & 741 & 43 & 43.9 & 93.0 & 93 & $2.2 \%$ \\
\hline p10 & 46 & 23 & 5.5 & 209.0 & 209 & 715 & 148 & 74.2 & 165.0 & 165 & $26.7 \%$ \\
\hline Ave. & 41.6 & & 5.3 & & & 609.6 & & 194.7 & & & $9.93 \%$ \\
\hline
\end{tabular}

Table 3: Performance evaluation of random topologies with 13 nodes in a square area of 1000 $m^{2}$, with parameters $P=0.2 \mathrm{~mW}, \gamma=10$, and $\eta=2 * 10^{-10} \mathrm{~mW}$. The time is in seconds.

\begin{tabular}{|l|rrrrrrrrrr|r|}
\hline & \multicolumn{5}{|c|}{ No Cooperation } & \multicolumn{5}{|c|}{ CoOperAtion } & \\
Ins. & $|\mathcal{L}|$ & Iter & Time & $L B_{1}$ & $U B_{1}$ & $|A|$ & Iter & Time & $L B_{2}$ & $U B_{2}$ & Gain \\
\hline p11 & 50 & 23 & 5.1 & 73.0 & 73 & 786 & 171 & 77 & 73.0 & 73 & $0.00 \%$ \\
p12 & 54 & 19 & 7.1 & 290.0 & 290 & 812 & 798 & 1288 & 236.3 & 237 & $22.36 \%$ \\
p13 & 50 & 34 & 12.4 & 232.0 & 232 & 696 & 1189 & 1085 & 215.3 & 216 & $7.41 \%$ \\
p14 & 60 & 23 & 11.3 & 148.0 & 148 & 1004 & 399 & 639 & 140.3 & 141 & $4.96 \%$ \\
p15 & 54 & 25 & 9.8 & 257.0 & 257 & 845 & 546 & 834 & 230.3 & 231 & $11.26 \%$ \\
p16 & 56 & 32 & 12.8 & 85.8 & 86 & 885 & 292 & 377 & 85.7 & 86 & $0.00 \%$ \\
p17 & 84 & 78 & 75.6 & 162.6 & 163 & 1396 & 736 & 7200 & 161.0 & 161 & $1.24 \%$ \\
p18 & 64 & 26 & 14.1 & 203.0 & 203 & 1020 & 1092 & 3561 & 189.5 & 190 & $6.84 \%$ \\
p19 & 74 & 19 & 11.8 & 225.0 & 225 & 1168 & 251 & 1304 & 207.0 & 207 & $8.70 \%$ \\
p20 & 62 & 37 & 17.7 & 236.3 & 237 & 974 & 610 & 1562 & 205.0 & 205 & $15.61 \%$ \\
\hline Ave. & 60.8 & & 17.8 & & & 958.6 & & 1793 & & & $7.84 \%$ \\
\hline
\end{tabular}


Table 4: Performance evaluation of random topologies with 13 nodes in a square area of 1000 $m^{2}$, with parameters $P=0.3 \mathrm{~mW}, \gamma=10$, and $\eta=2 * 10^{-10} \mathrm{~mW}$. The time is in seconds.

\begin{tabular}{|l|rrrrr|rrrrr|r|}
\hline & \multicolumn{7}{|c|}{ No-Cooperation } & \multicolumn{5}{|c|}{ Cooperation } & \\
Ins. & $\mathcal{L}$ & Iter & Time & $L B_{1}$ & $U B_{1}$ & $|A|$ & Iter & Time & $L B_{2}$ & $U B_{2}$ & Gain \\
\hline p21 & 70 & 38 & 26.79 & 150.5 & 151 & 1057 & 1365 & 7200 & 149 & 149 & $1.34 \%$ \\
p22 & 64 & 29 & 16.71 & 184 & 184 & 1014 & 1848 & 7061 & 166.4 & 167 & $10.18 \%$ \\
p23 & 52 & 31 & 11.48 & 230 & 230 & 820 & 1489 & 2026 & 203.5 & 204 & $12.75 \%$ \\
p24 & 80 & 40 & 41.37 & 186.22 & 187 & 1424 & 861 & 6479 & 183.2 & 184 & $1.63 \%$ \\
p25 & 80 & 22 & 16.16 & 200.5 & 201 & 1309 & 778 & 7200 & 193.5 & 194 & $3.61 \%$ \\
p26 & 68 & 36 & 29.21 & 181.75 & 182 & 1068 & 461 & 2169 & 177 & 177 & $2.82 \%$ \\
p27 & 98 & 42 & 30.88 & 161.33 & 162 & 1850 & 421 & 7200 & 154 & 154 & $5.19 \%$ \\
p28 & 82 & 28 & 20.41 & 162.68 & 163 & 1337 & 736 & 7200 & 162 & 162 & $0.62 \%$ \\
p29 & 92 & 34 & 25.27 & 198 & 198 & 1630 & 273 & 4083 & 191 & 191 & $3.66 \%$ \\
p30 & 78 & 18 & 12.54 & 209 & 209 & 1267 & 1176 & 7200 & 174.3 & 175 & $19.43 \%$ \\
\hline Ave. & 76.4 & 23.1 & \multicolumn{10}{|c|}{1277.6} & 5782 & & $6.12 \%$ \\
\hline
\end{tabular}

tage of cooperation is apparent. The figure reports results for networks with 15 nodes and the same technical parameters as before. This time, the traffic demands are generated in a specific way. The number of demands $|\mathcal{D}|$ grows from 5 to 20 . For each value of $|\mathcal{D}|$, the source and destination nodes of the demands are chosen with a fixed hop distance $h$, where $h=2,3,4,5,6$. In practice, for $h=6$, we have considered all the demands with hop distance being equal to or greater than 6 . The purpose is to analyze the behavior of cooperation not only as a function of traffic load, but also as a function of source-destination distance. The figure gives the percentage gain of using cooperation in the schedule length. For example, for the instances with 20 demands and $h \geq 6$, the schedule length by cooperation is $37 \%$ shorter than that without cooperation. The figure shows clearly that the higher is the source-destination distance, the higher will be the cooperation gain. This is due to the fact that in the cooperation graph the hop distances between pairs of nodes are usually smaller, because of the presence of super-links that give additional shortcuts. 


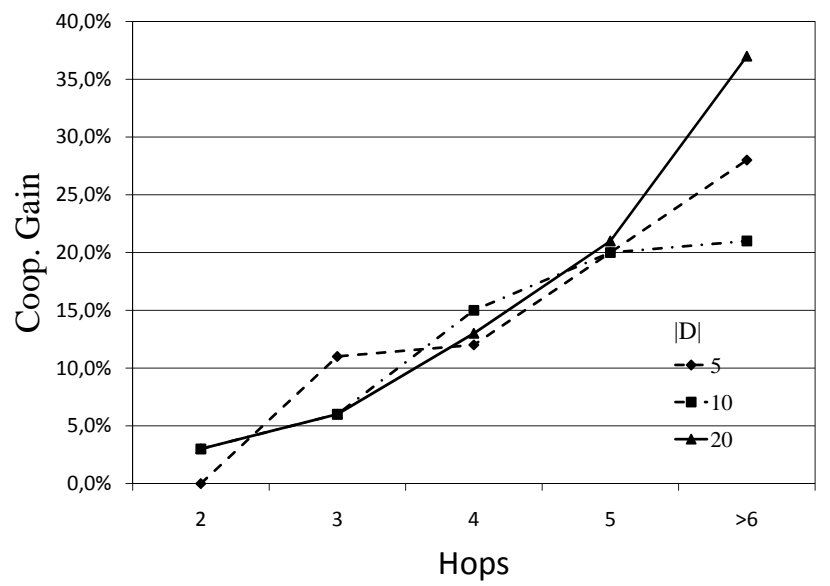

Figure 14: Cooperation gain versus the number of hops for different values of $|D|$.

\subsection{Additional Comments on the Results}

Recall that the proposed approach compares the optimal routing and scheduling for hop-by-hop forwarding and cooperative relaying (decode-and-forward). Therefore, the two mechanisms are compared considering their best achievable performance when the propagation parameters are all known. Since protocol overheads are not included, hop-by-hop forwarding becomes the special case of cooperative relaying where a single node is selected for each transmission. Thus the cooperation gain is always greater than or equal to zero.

The values of the cooperation gain we have obtained for some illustrative examples and a set of random instances point out that the advantage of cooperation is highly dependent on network topology and traffic pattern. In single source-destination pair scenarios, channel capacity improvements because of cooperation prevail. In networking scenarios, the reuse level of radio resources is a key element, and the results show that the limitation of cooperation on the reuse level is particularly severe when the network is dense and/or many traffic flows must be served in parallel. This is due to the high level of interference 
that makes cooperation less attractive and the fact that nodes can not be in multiple cooperation groups at the same time because of half duplex operation.

The results yields another insight that could not be observed with classical schemes that consider two-steps (hops) cooperation only. Namely, the cooperation gain increases remarkably with the number of hops traversed by traffic flows. This is because a large hop distance leads to a higher degree of freedom in optimizing scheduling and routing, and the advantage of increased transmission range by cooperation becomes more apparent.

\section{Conclusions}

In this paper we have proposed an optimization approach for evaluating the resource efficiency of cooperative relaying vis-a-vis conventional multi-hop forwarding. We have modeled interference through the physical model based on SINR values, and considered the decode-and-forward cooperation scheme that defines a cooperation graph. Considering a spatial time division multiplexing framework, we have designed a methodology for optimizing scheduling and routing both in case of cooperative relay and traditional multi-hop forwarding. The resource efficiency has been measured considering the minimum number of time slots required to serve all traffic demands.

The proposed methodology extends previous works in allowing for evaluating the efficiency of cooperation in networks with arbitrary topology, where the interference is not just statistically characterized but controlled through the scheduling of simultaneous transmissions. Moreover, our approach provides a tool for managing in an efficient way the radio resource in networks of cooperative nodes through joint routing and scheduling optimization.

The results presented show that the overall efficiency greatly depends on the network topology and the traffic pattern. The limitation of cooperation on improving the reuse level of radio resources is particularly apparent when the network is dense and a large number of traffic flows must be served. For scenarios where the underlying network has many direct links as well as source- 
destination pairs, the gain of cooperation tends to diminish. On the other hand, the advantage of cooperation in a networking context is significant as the number of hops increases.

\section{Acknowledgments}

We are grateful to Lazzaro Baj for his contribution to the experimental evaluation. The work of the first two authors has been partially supported by project PRIN SESAME of the Italian ministry of university and research, and the European Network of Excellence EURO-NF. The work of the last author has been partially supported by the Swedish ELLIIT network. We thank the anonymous reviewers for their valuable comments and suggestions

\section{References}

[1] M. Abolhasan, T. Wysocki, E. Dutkiewicz, A review of routing protocols for mobile ad hoc networks, Ad Hoc Networks, vol. 2, no. 1, 2004.

[2] M. Gastpar, M. Vetterli, On the capacity of wireless networks: the relay case, in Proc. of IEEE INFOCOM, New York, US, 2002.

[3] A. Sendonaris, E. Erkip, B. Aazhang, User cooperation diversity, Part I: System description/Part II: Implementation aspects and performance analysis, IEEE Trans. on Communications, vol. 51, no. 11, 2003, pp 1927-1948.

[4] E.C. van der Meulen, Three-terminal communication channels, Advanced Appliled Probability, vol. 3, 1971, pp. 120-154.

[5] T.M. Cover, A.A.E. Gamal, Capacity theorems for the relay channel, IEEE Trans. on Information Theory, vol. 25, no. 5, 1979, pp. 572-584.

[6] Dohler, F. Said, H. Aghvami, Concept of virtual antenna arrays, in Proc. of IEEE Globecom 2002, Taipei, Taiwan, 2002.

[7] M. Dohler, A. Gkelias, H. Aghvami, 2-Hop distributed MIMO communication system, IEEE Electronics Letters, 2003.

[8] J.N. Laneman, D.N.C. Tse, and G.W. Wornell, Cooperative Diversity in Wireless Networks: Efficient Protocols and Outage Behavior, IEEE Trans. on Information Theory, vol. 50, no. 12, Dec. 2004, pp. 3062-3080.

[9] P. Herhold, E. Zimmermann, G. Fettweis, Cooperative multi-hop transmission in wireless networks, Computer Networks, vol. 49, 2005, pp. 299-324.

[10] M. Gastpar, G. Kramer, P. Gupta, The multiple-relay channel: coding and antennaclustering capacity, in Proc. of IEEE Int. Symp. on Information Theory, Lausanne, Switzerland, 2002. 
[11] A. Hst-Madsen and J. Zhang, Capacity Bounds and Power Allocation for Wireless Relay Channels, IEEE Trans. on Information Theory, vol. 51, no. 6, pp. 2020-2040, June 2005.

[12] G. Kramer, M. Gastpar, and P. Gupta, Cooperative Strategies and Capacity Theorems for Relay Networks, IEEE Trans. on Information Theory, vol. 51, no. 9, pp. 3037-3063, Sept. 2005.

[13] A. Stefanov, E. Erkip, Cooperative coding for wireless networks, in Proc. of IEEE Conference on Mobile Wireless Communication Networks, Stockholm, Sweden, 2002.

[14] M.C. Valenti, B. Zhao, Distributed turbo coded diversity for the relay channel, IEE Electronics Letters, vol. 39, no. 10, 2003, pp. 786-787.

[15] M.C. Valenti, B. Zhao, Distributed turbo codes: towards the capacity of the relay channel, in Proc. of IEEE VTC 2003.

[16] B. Zhao and M.C. Valenti, Practical Relay Networks: A Generalization of Hybrid-ARQ, IEEE Journal on Selected Areas in Comm., vol. 23, no. 1, Jan. 2005, pp. 7-18.

[17] X. Deng and A.M. Haimovich, Power Allocation for Cooperative Relaying in Wireless Networks, IEEE Communications Letters, vol. 9, no. 11, Nov. 2005, pp. 994-997.

[18] S. Serbetli and A. Yener, Optimal Power Allocation for Relay Assisted F/TDMA Ad Hoc Networks, in Proc. of Int. Conf. on Wireless Networks, Communications and Mobile Computing, 2005.

[19] J. Boyer, D.D. Falconer, H. Yanikomeroglu, Multihop Diversity in Wireless Relaying Channels, IEEE Trans. on Communications, vol. 52, no. 10, 2004, pp. 1820-1830.

[20] K. Azarian, H.E. Gamal, and P. Schniter, On the Achievable Diversity-Multiplex Tradeoff in Half-Duplex Cooperative Channels, IEEE Trans. on Information Theory, vol. 51, no. 12 , Dec. 2005, pp. 4152-4172.

[21] T. Abe, H. Shi, T. Asai, and H. Yoshino, ARelaying Scheme for MIMO Wireless Networks with Multiple Source and Destination Pairs, in Proc. of 11th Asia-Pacific Conference on Communications (APCC), 2005.

[22] G. Li and H. Liu, On the Capacity of Broadband Relay Networks, in Proc. of 38th Asilomar Conference on Signals, Systems and Computers, 2004.

[23] G. Scutari, S. Barbarossa, and D. Ludovici, Cooperation Diversity in Multihop Wireless Networks Using Opportunistic Driven Multiple Access, in Proc. of IEEE Workshop on Signal Processing Advances in Wireless Comm. (SPAWC03), June 2003.

[24] A. Bletsas, H. Shin, and M. Z. Win. Cooperative communications with outage-optimal opportunistic relaying. IEEE Trans. Wireless Commun., 6(9):3450-3460, Sept. 2007.

[25] M. Qin and R. Blum, Capacity of Wireless Ad Hoc Networks with Cooperative Diversity: A Warning on the Interaction of Relaying and Multi-Hop Routing, in Proc. of IEEE ICC 2005.

[26] P. Gupta, P.R. Kumar, The capacity of wireless networks, IEEE Trans. on Information Theory, vol. 46, no. 2, 2000, pp. 388-404. 
[27] A.H. Madsen, Capacity Bounds for Cooperative Diversity, IEEE Trans. on Information Theory, vol. 52, no. 4, 2006, pp. 1522-1544.

[28] I. Krikidis, J.S. Thompson, S. McLaughlin, N. Goertz, Max-Min Relay Selection for Legacy Aplify-and-Forward Systems with Interference, IEEE Trans. on Wireless Communications, vol. 8, no. 6, 2009, pp. 3016-3027.

[29] Y. Zhu, H. Zheng, Understanding the Impact of Interference on Collaborative Relays, IEEE Trans. on Mobile Computing, vol. 7, no. 6, June 2008, pp. 724-736.

[30] P. Björklund, P. Värbrand, Di Yuan, A Column Generation Method for Spatial TDMA Scheduling in Ad Hoc Networks, Ad Hoc Networks, vol. 2, no. 4, October 2004, pp. $405-418$.

[31] D. M. Blough, S. Das, G. Resta, P. Santi, A Framework for Joint Scheduling and Diversity Exploitation under Physical Interference in Wireless Mesh Networks, in Proc. of IEEE MASS 2008.

[32] A. Capone, G. Carello, I. Filippini, S. Gualandi, F. Malucelli, Solving a Resource Allocation Problem in Wireless Mesh Networks: a Comparison Between a CP-based and a Classical Column Generation, Networks, vol. 55, no. 3, 2010, pp. 221-233.

[33] A. Capone, G. Carello, I. Filippini, S. Gualandi, and F. Malucelli, Routing, Scheduling and Channel Assignment in Wireless Mesh Networks: Optimization Models and Algorithms. Ad Hoc Networks, vol. 8, no. 6, 2010, pp. 545-563.

[34] L.A. Wolsey, Integer Programming. John Wiley \& Sons, New York, 1998.

[35] H.P. Williams, Model Building in Mathematical Programming. Wiley, 3rd edition, 1995. 